\title{
Could MAO-B Inhibitor Withdrawal Rather than Nilotinib Benefit Explain the Dopamine Metabolite Increase in Parkinsonian Study Subjects?
}

\section{Dear Editors,}

The recent study by Pagan and colleagues [1] followed 12 subjects with Parkinson's disease or dementia with Lewy bodies before and while taking nilotinib for up to 24 weeks. Although this pilot trial was open-label and had no control group as appropriately highlighted by the authors, the hope that the clinical improvements noted in most of the subjects could be an effect of nilotinib was buoyed by neurochemical changes in their CSF. These indeed ought to be much less susceptible to placebo effects. In presenting their most noteworthy biomarker findings the authors reported, "CSF levels of homovanillic acid [HVA] are significantly increased between baseline and 24 weeks of treatment." HVA is the main CSF metabolite of dopamine in humans, and its increase in this study was interpreted by the authors as supporting a hypothetical protective effect of nilotinib on dopaminergic neurons possibly via mechanisms involving alpha-Synuclein or Parkin. The observed HVA increase over time and an inference of improved dopamine production while on nilotinib have been widely cited in institutional announcements and media reports as evidence that the clinical improvements in this study could represent true benefits of nilotinib treatment on dopaminergic neuron function.

However, a simple alternative interpretation of the HVA increase should be considered based on the withdrawal of a monoamine oxidase-B (MAO-B) inhibitor from most ( 8 of 11) of the subjects (due to increased psychosis) less than a month after the baseline CSF collection. Because MAO-B catalyzes the oxidative metabolism of dopamine to HVA, discontinuation of an MAO-B inhibitor is expected to raise CSF HVA levels [2, 3] as has been clearly demonstrated in Parkinson's disease patients [4]. Of note although rasagiline is an irreversible inhibitor of MAO-B the enzyme's activity is expected to revert to near normal within 8 weeks of discontinuation, $[4,5]$ - well within the $\sim 20$ week period subjects had apparently been off of or on a reduced dose of rasagiline before their final lumbar puncture. Accordingly, it seems likely that the partial or complete withdrawal of MAO-B inhibitor contributed to the observed HVA increase in this nilotinib study.

Although stratifying HVA analysis by rasagiline dose reduction could help reconcile these alternatives if data are available, the very small sample size of this pilot study may limit interpretation. Nevertheless, it may be valuable in any larger, future study attempting to replicate these findings to consider whether any HVA increase correlates with MAO-B inhibitor reduction.

Sincerely,

Michael A. Schwarzschild, MD PhD

MassGeneral Institute for Neurodegenerative Disease

Boston, Massachusetts, USA

E-mail: michaels@helix.mgh.harvard.edu.

\section{REFERENCES}

[1] Pagan F, Hebron M, Valadez EH, Torres-Yaghi Y, Huang X, Mills RR, Wilmarth BM, Howard H, Dunn C, Carlson A, Lawler A, Rogers SL, Falconer RA, Ahn J, Li Z, \& Moussa C 
(2016) Nilotinib Effects in Parkinson's disease and Dementia with Lewy bodies. J Parkinsons Dis, 6, 503-517.

[2] Sunderland T, Tariot PN, Cohen RM, Newhouse PA, Mellow AM, Mueller EA, \& Murphy DL (1987) Dose-dependent effects of deprenyl on CSF monoamine metabolites in patients with Alzheimer's disease. Psychopharmacology, 91, 293-296.

[3] Martignoni E, Bono G, Blandini F, Sinforiani E, Merlo P, \& Nappi G (1991) Monoamines and related metabolite levels in the cerebrospinal fluid of patients with dementia of Alzheimer type. Influence of treatment with L-deprenyl. $J$ Neural Transm Park Dis Dement Sect, 3, 15-25.
[4] Parkinson Study Group (1995) Cerebrospinal fluid homovanillic acid in the DATATOP study on Parkinson's disease. Arch Neurol, 52, 237-243.

[5] Fowler JS, Volkow ND, Logan J, Wang GJ, MacGregor RR, Schyler D, Wolf AP, Pappas N, Alexoff D, Shea C, et al. (1994) Slow recovery of human brain MAO B after L-deprenyl (Selegeline) withdrawal. Synapse, 18, 86-93. 